- Original Article

\title{
The Effects of Alcohol on Quality of Sleep
}

\author{
Soon-Yeob Park', Mi-Kyeong Oh ${ }^{1, *}$, Bum-Soon Lee' ${ }^{1}$ Haa-Gyoung Kim', Won-Joon Lee', Ji-Ho Lee', Jun-Tae Lim', \\ Jin-Young Kim²
}

'Department of Family Medicine, Gangneung Asan Hospital, University of Ulsan, College of Medicine, Gangneung, Korea

${ }^{2}$ Department of Family Medicine, Chungbuk National University Hospital, Cheongju, Korea

Background: Alcohol is traditionally known to have a relaxing effect. However, persons who consume alcohol in excessive amounts suffer from poor sleep quality and patients with alcohol use disorders commonly report insomnia. In this study, we aimed to evaluate the effects of alcohol use on sleep quality.

Methods: A questionnaire-based cross-sectional survey was conducted with 234 men and 159 women who had visited a general hospital. We used structured questionnaires, including Alcohol Use Disorder Identification TestKorean revised version (AUDIT-KR) and the Pittsburgh Sleep Quality Index-Korean version (PSQI-K). We analyzed the association between scores for all subcategories of the PSQI-K and the AUDIT-KR and then analyzed the correlation between AUDIT-KR and global PSQI-K scores.

Results: The global PSQI-K score for men was positively correlated with the AUDIT-KR score ( $\mathrm{P}=0.008)$ after adjusting for age, chronic disease, tobacco use, exercise, depression, and anxiety. The AUDIT-KR score was significantly associated with subjective sleep quality $(\mathrm{P}=0.005)$, sleep duration $(\mathrm{P}=0.047)$, and sleep disturbance $(\mathrm{P}=0.048)$; it was not associated with sleep latency, sleep efficiency, or daytime dysfunction. Sleep disturbances due to snoring were significantly associated with total AUDIT-KR score $(\mathrm{P}=0.008)$. There was no correlation between the global PSQI-K and AUDIT-KR scores for women $(\mathrm{P}=0.333)$. However, daytime dysfunction showed a significant association with total AUDIT-KR score $(\mathrm{P}=0.048)$.

Conclusion: Men with higher AUDIT-KR scores tended to suffer from poor sleep quality. AUDIT-KR scores showed significant correlations with subjective sleep quality, sleep duration, and sleep disturbances in men.

Keywords: Alcohol Drinking; Sleep Disorders; Sleep Quality 


\section{INTRODUCTION}

Sleep is commonly known to facilitate recovery from physical, mental, and brain fatigue caused by daytime activities. Sleep allows the human body the rest time required for memory and learning, preservation of energy, development, and growth. ${ }^{1)}$ Recently, several studies on sleep have reported that poor quality sleep not only leads to increased stress, fatigue, and mood disorders ${ }^{2)}$ but also raises risks of obesity, metabolic syndrome, and cardiovascular disease. ${ }^{3)}$ Conversely, many diseases also affect sleep quality. Depression, anxiety, musculoskeletal disorders, obesity, restless leg syndrome, and other chronic diseases are all known to cause sleep disorders. ${ }^{4-8)}$

Moderate alcohol consumption is known to decrease the risk of mortality, ${ }^{9-11)}$ while excessive drinking can increase the onset of various diseases and lead to increased risk of mortality. ${ }^{912-15)}$ Excessive drinking also cause a range of problems with the nervous system; such problems include memory disorders, movement disorders, peripheral neuropathy, and reduced learning ability. ${ }^{16,17)}$ As of 2012, the rate of high-risk drinking (average alcohol consumption of $7+$ drinks on $2+$ days per week) among Korean adults males is $21.8 \%$. The high-risk drinking rate exceeds $25 \%$ for those aged $30-59$, indicating that a significant part of the population is exposed to drinking related dangers. Among women, on the other hand, the high-risk drinking rate has been reported at $6.0 \%{ }^{18)}$

Alcohol consumption also affects sleep quality in various ways. A number of studies have shown that drinking momentarily increases sleepiness, but later causes frequent nighttime and early morning awakenings. ${ }^{19)}$ Individuals with alcohol use disorders frequently consume alcohol before sleep in an effort to improve their sleep. Many moderate alcohol users also drink before sleep if they suffer from insomnia. On the contrary, other studies have shown no correlation between drinking patterns and sleep quality. ${ }^{4,20)}$ While it is believed that drinking patterns are significantly related to sleep patterns, no empirical correlation has been confirmed to date and studies on the correlation between drinking patterns and sleep quality of Koreans are virtually non-existent.

Therefore, we conducted this study to examine the effects of alcohol consumption on sleep quality and to provide recommendations for improving sleep quality.

\section{METHODS}

\section{Subjects}

This study was conducted on patients, and their guardians, who visited Gangneung Asan Hospital in Gangneung, Korea from January 2014 to July 2014. Among 560 adults who voluntarily completed the informed consent process, subjects diagnosed with conditions known to affect sleep, including life-thre- atening critical diseases, depression, anxiety, restless leg syndrome, and sleep apnea were excluded. Thus, we selected 234 men and 159 women who sincerely answered study questionnaires. This study was conducted after being reviewed and approved by the hospital's institutional review board (GNAH IRB 2014-017).

\section{Survey Methods}

\section{1) Structured questionnaire}

In order to assess basic subject characteristics, a questionnaire was administered asking about a subject's age, gender, and medical history (e.g., hypertension, diabetes, dyslipidemia, chronic musculoskeletal disease, etc.). In order to identify smoking patterns, subjects were categorized as current smokers, former smokers, or non-smokers. Subjects were also asked about their exercise habits, including total number and duration of exercise sessions per week. This data was used to categorize subjects into one of three exercise levels: those exercising less than once per week, those exercising 1-2 times per week, and those exercising 3 times or more per week.

Although persons with a history of depression or anxiety were excluded from the study, we used a depression and anxiety screening tool, which has been standardized in Korea; this tool was used in order to correct for the role of depression or anxiety that are known to affect sleep quality. More specifically, we used the 'two-question depression test' and those who answered yes to one or both of the questions were assumed to be experiencing some level of depression. ${ }^{21)}$ We also asked subjects four questions covering primary symptoms of anxiety, adapted from the Korean-translated Goldberg Anxiety Scale (GAS). Those who answered yes to two or more of the four questions were assumed to be experiencing some level of anxiety. ${ }^{22)}$

\section{2) Alcohol drinking state assessment questionnaire}

In order to investigate alcohol consumption rates, we used the Alcohol Use Disorder Identification Test-Korean revised version (AUDIT-KR), a tested and validated Korean version of the AUDIT that is widely used for assessment of alcohol use disorders and hazardous drinking. ${ }^{23)}$ The AUDIT-KR consists of 10 questions, with each question rated on a 4-point scale. The higher the respondent's alcohol dependency, the higher the total score. The maximum possible score is 40 points. The cutoff value for alcohol use disorders is 10 points for men and 8 points for women.

\section{3) Sleep quality assessment questionnaire}

In order to evaluate subjects' sleep quality, we used the Pittsburgh Sleep Quality Index-Korean version (PSQI-K), a tested and validated Korean-language version of the Pittsburgh Sleep Quality Index (PSQI), which has been widely used as a sleep quality assessment tool since its translation in $2012 .^{24)}$ We in- 
vestigated the scores of the 7 components of the PSQI-K that affect sleep quality: subjective sleep quality, sleep duration, sleep latency, habitual sleep efficiency, sleep disturbance, use of sleep medication, and daytime dysfunction. The global score of the PSQI-K is the sum of all component scores. The minimum possible score is 0 , indicating ideal sleep quality, and the maximum possible score is 21 , indicating poor sleep quality. Subjects with total scores of 5 and below were defined as 'good sleepers,' and those with total scores of 6 and above were defined as 'poor sleepers.' The 7 component scores are rated on a 3-point ascending scale, with 0 points indicating ideal sleep quality and 3 points indicating poor sleep quality. Each component score of $0-1$ points was determined as having good effects on sleep quality, while scores of 2-3 points were determined as having bad effects on sleep quality.

\section{Statistical Analysis}

In this study, we used PASW SPSS Statistics ver. 18.0 (SPSS Inc., Chicago, IL, USA) for statistical analyses with significance level set at less than 0.05 for all statistical operations. We performed chi-square tests and independent sample t-tests to assess gender differences in initial reports of underlying disease, smoking status, exercise frequency, depressive symptoms, and anxiety symptoms. We used the Wilcoxon two-sample test to assess gender differences in AUDIT-KR scores. To investigate the relationship between global or component scores of the PSQI-K and AUDIT-KR scores, we performed the Mann-Whitney test.

In order to analyze the correlation between AUDIT-KR scores and PSQI-K scores, we performed a multiple linear regression analysis adjusting for age, chronic disease, current smoking status, regular exercise, two-question depression test scores, and GAS scores. Any subjects diagnosed with hypertension, diabetes, ischemic heart disease, stroke, or chronic musculoskeletal diseases and undergoing treatment at the hospital for any of these conditions were defined as having chronic disease, for the purposes of this study. Subjects who reported that they exercised 3 times or more per week were defined as regular exercisers.

\section{RESULTS}

\section{Baseline Characteristics of Subjects}

The average age of the subjects was $51.1 \pm 12.0$ years for men and $48.6 \pm 10.0$ years for women. The number of subjects categorized as having chronic disease was significantly greater among men as compared to women $(\mathrm{P}=0.001)$. As for smoking status, the number of current smoker and former smokers were significantly greater among men than women $(\mathrm{P}<0.001)$. Reported frequency of weekly exercise was also significantly greater among men than women in this study $(\mathrm{P}=0.004)$.

Total AUDIT-KR scores were significantly higher among men
Table 1. Baseline characteristic of study subjects

\begin{tabular}{|c|c|c|c|}
\hline Variable & Men $(n=234)$ & Women $(n=159)$ & P-value* \\
\hline Age (y) & $51.1 \pm 12.0$ & $48.6 \pm 10.0$ & 0.075 \\
\hline \multicolumn{4}{|l|}{ Underlying disease } \\
\hline Hypertension & $83(35.5)$ & $32(20.1)$ & 0.001 \\
\hline Diabetes & 27 (11.5) & $7(4.4)$ & 0.014 \\
\hline Ischemic heart disease & $12(5.1)$ & $3(1.9)$ & 0.100 \\
\hline Stroke & $1(0.4)$ & $4(2.5)$ & 0.070 \\
\hline Rheumatic disease & $18(7.7)$ & $11(6.9)$ & 0.773 \\
\hline Chronic disease & $112(47.9)$ & 49 (30.8) & 0.001 \\
\hline Smoking status & & & $<0.001$ \\
\hline Current smoker & 67 (28.6) & $3(1.9)$ & \\
\hline Ex-smoker & $114(48.7)$ & $6(3.8)$ & \\
\hline Non-smoker & $53(22.6)$ & $150(94.3)$ & \\
\hline Exercise & & & 0.004 \\
\hline None & $96(41.0)$ & $91(57.2)$ & \\
\hline 1-2 times a week & 87 (37.2) & $48(30.2)$ & \\
\hline At least 3 times a week & $51(21.8)$ & $20(12.6)$ & \\
\hline Depressive mood & $64(27.4)$ & 49 (30.8) & 0.456 \\
\hline Anxious mood & $33(14.1)$ & $23(14.5)$ & 0.920 \\
\hline AUDIT-KR & $9[3,16]^{\dagger}$ & $1[0,3]^{\dagger}$ & $<0.001^{\ddagger}$ \\
\hline Alcohol use disorder ${ }^{\S}$ & & & $<0.001$ \\
\hline No & $120(51.3)$ & $141(88.7)$ & \\
\hline Yes & $114(48.7)$ & $18(11.3)$ & \\
\hline $\begin{array}{l}\text { Pittsburgh Sleep Quality } \\
\text { Index-Korean version }\end{array}$ & $5[3,7]^{\dagger}$ & $4[3,6]^{\dagger}$ & $0.033^{\ddagger}$ \\
\hline Good sleeper (score $\leq 5)(\%)$ & $136(58.1)$ & $105(66.0)$ & 0.114 \\
\hline Poor sleeper (score >5) (\%) & 98 (41.9) & $54(34.0)$ & \\
\hline
\end{tabular}

Values are presented as mean \pm standard deviation or number (\%).

AUDIT-KR, Alcohol Use Disorder Identification Test-Korean Revised.

*By independent sample t-test or chi-square test. 'Values are presented as median [25 percentile, 75 percentile]. 讘 Wilcoxon two-sample test. ${ }^{\circledR}$ Alcohol use disorder is indicated by an of AUDIT-KR cutoff value greater than 10 points in men and 8 points in women.

than women $(\mathrm{P}<0.001)$ and the number of subjects categorized as having an alcohol use disorder was also significantly greater among males than females $(\mathrm{P}<0.001)$. According to results of the depression and anxiety screening tests, there were no significant gender differences between subjects with symptoms of depression and those with symptoms of anxiety. While global PSQI-K score was significantly higher among men than women $(\mathrm{P}=0.033)$, when subjects were categorized by sleep quality (good sleepers and poor sleepers) the ratio of poor sleepers became non-significant $(\mathrm{P}=0.114)$ (Table 1).

\section{Relationship between Each Component of Sleep Quality and Alcohol Use Disorder Identification Test-Korean Revised Version Score}

When our subjects were categorized by sleep quality, based upon their total PSQI-K scores, there were no significant differences between the two groups and AUDIT-KR scores for either men or women. Examination of the correlation between each PSQI-K component score and AUDIT-KR score among men, revealed that the group with poor subjective sleep quality showed significantly higher AUDIT-KR scores than the group with good subjective sleep quality $(\mathrm{P}=0.005)$. Additionally, subjects with short sleep duration ( $<6$ hours of sleep per night on aver- 
Table 2. Relationship between component score of PSQI-K and AUDIT-KR

\begin{tabular}{|c|c|c|c|c|c|c|}
\hline \multirow{2}{*}{ Component score of PSQI-K } & \multicolumn{3}{|c|}{ Men } & \multicolumn{3}{|c|}{ Women } \\
\hline & No. & AUDIT-KR & P-value ${ }^{*}$ & No. & AUDIT-KR & P-value ${ }^{*}$ \\
\hline \multicolumn{7}{|l|}{ Total PSQI-K score } \\
\hline Good sleeper (total score $\leq 5$ ) & 136 & $9.21 \pm 7.62$ & \multirow[t]{2}{*}{0.105} & 105 & $2.45 \pm 4.15$ & \multirow[t]{2}{*}{0.333} \\
\hline Poor sleeper (total score >5) & 98 & $11.53 \pm 9.42$ & & 54 & $3.74 \pm 6.10$ & \\
\hline \multicolumn{7}{|l|}{ Subjective sleep quality } \\
\hline Good & 162 & $9.04 \pm 7.81$ & \multirow[t]{2}{*}{0.005} & 125 & $2.78 \pm 4.63$ & \multirow[t]{2}{*}{0.674} \\
\hline Poor & 72 & $12.74 \pm 9.29$ & & 34 & $3.29 \pm 5.93$ & \\
\hline \multicolumn{7}{|l|}{ Sleep latency } \\
\hline$\leq 30 \min$ & 179 & $9.99 \pm 8.21$ & \multirow[t]{2}{*}{0.77} & 116 & $2.98 \pm 4.64$ & \multirow[t]{2}{*}{0.075} \\
\hline$>30 \mathrm{~min}$ & 55 & $10.78 \pm 9.36$ & & 43 & $2.63 \pm 5.64$ & \\
\hline \multicolumn{7}{|l|}{ Sleep duration } \\
\hline$\geq 6 \mathrm{~h}$ & 207 & $9.67 \pm 8.04$ & \multirow[t]{2}{*}{0.047} & 145 & $2.81 \pm 4.79$ & \multirow[t]{2}{*}{0.305} \\
\hline$<6 \mathrm{~h}$ & 27 & $14.07 \pm 10.71$ & & 14 & $3.64 \pm 6.30$ & \\
\hline \multicolumn{7}{|l|}{ Sleep efficiency ${ }^{\dagger}$} \\
\hline$\geq 75 \%$ & 207 & $9.74 \pm 8.11$ & \multirow[t]{2}{*}{0.094} & 148 & $2.84 \pm 4.79$ & \multirow[t]{2}{*}{0.574} \\
\hline$<75 \%$ & 27 & $13.56 \pm 10.48$ & & 11 & $3.45 \pm 6.65$ & \\
\hline \multicolumn{7}{|l|}{ Sleep disturbance } \\
\hline No & 197 & $9.52 \pm 7.84$ & \multirow[t]{2}{*}{0.048} & 136 & $2.74 \pm 4.74$ & \multirow[t]{2}{*}{0.422} \\
\hline Yes & 37 & $13.68 \pm 10.76$ & & 23 & $3.74 \pm 5.91$ & \\
\hline \multicolumn{7}{|l|}{ Daytime dysfunction } \\
\hline No & 169 & $9.87 \pm 8.21$ & \multirow[t]{2}{*}{0.445} & 122 & $2.25 \pm 3.93$ & \multirow[t]{2}{*}{0.048} \\
\hline Yes & 65 & $10.98 \pm 9.16$ & & 37 & $4.97 \pm 6.96$ & \\
\hline \multicolumn{7}{|l|}{ Snoring ${ }^{\ddagger}$} \\
\hline$<1$ time/wk & 167 & $9.20 \pm 8.15$ & \multirow[t]{2}{*}{0.008} & 141 & $2.87 \pm 4.91$ & \multirow[t]{2}{*}{0.966} \\
\hline$\geq 1$ time/wk & 67 & $12.55 \pm 8.89$ & & 18 & $2.83 \pm 5.19$ & \\
\hline
\end{tabular}

Values are presented as mean \pm standard deviation.

PSQI-K, Pittsburgh Sleep Quality Index-Korean version; AUDIT-KR, Alcohol Use Disorder Identification Test-Korean Revised version.

*By Mann-Whitney test. ${ }^{\dagger}$ Hours asleep/hours in bed $\times 100$. ${ }^{\ddagger}$ Nighttime awakenings due to snoring.

age) showed significantly higher AUDIT-KR scores than those with longer sleep duration $(\mathrm{P}=0.047)$. Among men, those with more sleep disturbances showed significantly higher AUDITKR scores than those with fewer sleep disturbances $(\mathrm{P}=0.048)$. No significant differences in the correlation between AUDITKR scores were noted for sleep latency, sleep efficiency, and daytime dysfunction components. Although there was no statistical significance among individual sleep efficiency items, the group with lower efficiency tended to have higher AUDITKR scores as compared to the group with higher efficiency $(\mathrm{P}=$ 0.09). Subjects waking up from sleep, due to snoring, at least once per week showed significantly higher AUDIT-KR scores than other subjects $(\mathrm{P}=0.008)$ (Table 2$)$.

Among women, those with more daytime dysfunction showed significantly higher AUDIT-KR scores than those with lower daytime dysfunction $(\mathrm{P}=0.048)$, but the differences in AUDITKR scores were insignificant in relation to other components (Table 2).

\section{Multiple Linear Regression Analysis between Alcohol Use Disorder Identification Test-Korean Revised Version Scores and Sleep Quality Scores}

When a linear regression analysis was performed on the correlation between male AUDIT-KR and PSQI-K scores, a significant positive correlation was found when the factors affecting
Table 3. Multiple linear regression analysis of sleep quality by global Pittsburgh Sleep Quality Index-Korean version score

\begin{tabular}{|c|c|c|c|c|}
\hline \multirow{2}{*}{ Variable } & \multicolumn{2}{|c|}{ Men } & \multicolumn{2}{|c|}{ Women } \\
\hline & $\beta^{*}$ & P-value & $\beta^{*}$ & P-value \\
\hline \multicolumn{5}{|l|}{ Model 1} \\
\hline AUDIT-KR & 0.215 & 0.001 & 0.110 & 0.166 \\
\hline \multicolumn{5}{|l|}{ Model 2} \\
\hline AUDIT-KR & 0.222 & 0.001 & 0.071 & 0.411 \\
\hline Age & 0.003 & 0.969 & -0.150 & 0.142 \\
\hline Chronic disease & -0.049 & 0.482 & 0.153 & 0.112 \\
\hline Current smoker & -0.004 & 0.955 & & \\
\hline Regular exercise $^{\dagger}$ & -0.122 & 0.062 & -0.114 & 0.154 \\
\hline \multicolumn{5}{|l|}{ Model 3} \\
\hline AUDIT-KR & 0.171 & 0.008 & 0.014 & 0.861 \\
\hline Age & -0.026 & 0.707 & -0.104 & 0.264 \\
\hline Chronic disease & -0.028 & 0.676 & 0.096 & 0.271 \\
\hline Current smoker & 0.010 & 0.871 & & \\
\hline Regular exercise $^{\dagger}$ & -0.088 & 0.181 & -0.167 & 0.083 \\
\hline Depressive mood & 0.175 & 0.008 & 0.258 & 0.001 \\
\hline Anxious mood & 0.175 & 0.009 & 0.323 & $<0.001$ \\
\hline
\end{tabular}

PSQI-K, Pittsburgh Sleep Quality Index-Korean version; AUDIT-KR, Alcohol Use Disorder Identification Test-Korean revised version.

*Standard regression coefficient. ${ }^{\dagger} 3$ or more exercise sessions per week.

sleep were not corrected $(\mathrm{P}=0.001)$. A significant correlation was found, even after correcting for age, chronic disease, current smoking status, regular exercise, depression symptoms, and anxiety symptoms $(\mathrm{P}=0.008)$. Depression symptoms and anxiety symptoms were also positively correlated with PSQI-K 
scores $(\mathrm{P}=0.008$ and $\mathrm{P}=0.009$, respectively) (Table 3 ).

Among women, no significant correlation was found between AUDIT-KR and PSQI-K scores. However, depression symptoms and anxiety symptoms were found to be significantly and positively correlated with PSQI-K score $(\mathrm{P}=0.001$ and $\mathrm{P}<0.001$, respectively) (Table 3).

\section{DISCUSSION}

In the present study, which focused on the effects of alcohol consumption on sleep quality among adults aged 20 years and older, we found that AUDIT-KR and PSQI-K scores were significantly correlated among male subjects. In particular, we learned that alcohol consumption patterns are related to subjective sleep quality, sleep duration, and sleep continuation. In contrast, sleep latency was not correlated with alcohol consumption level. In other words, it appears that men with more alcohol consumption suffer from overall poor sleep quality, experience difficulty in maintaining sleep rather than falling asleep, suffer from shorter overall sleep duration, and therefore have worse subjective sleep quality. Among factors that disturb sleep, it was learned that snoring, in particular, is linked with alcohol consumption.

Among female subjects, AUDIT-KR and PSQI-K scores were not correlated. Perhaps this is because the number of female hazardous drinkers was notably lower than among male subjects and, therefore, the number of subjects with sleep disorder caused by alcohol might have been too low to yield any statistically significant result. When examining the correlation between the subcategories of the PSQI-K and the AUDIT-K among women, daytime dysfunction was shown to be significantly correlated with the AUDIT-K; this finding provides a basis for the conclusion that alcohol does not affect nighttime sleep duration among women as greatly as it deteriorates the recovery functions of sleep leading to daytime dysfunction.

Among both men and women, depression symptoms and anxiety symptoms were significantly correlated with increased PSQI-K values. In this study, we found that anxiety symptoms were more strongly correlated with PSQI-K values than depression symptoms and that women showed greater correlation between PSQI-K values and anxiety or depression symptoms as compared to men.

The correlation between alcohol consumption and sleep quality among men in this study was consistent with results previously reported by Roehrs and Roth ${ }^{25)}$ that alcohol generally disturbs sleep. ${ }^{19,26)}$ Chang et al. ${ }^{4)}$ and Vinson et al. ${ }^{20)}$ found that drinking does not have significant effects on sleep quality. However, these two studies did not control for gender and it could be posited that women act as an effect modifier. In our study, drinking did not significantly impact sleep quality among women likely because of generally low rates of alcohol consumption. It follows that women with problematic alcohol consumption experienced poorer sleep quality, similar to trends observed among men. ${ }^{26)}$

In a laboratory study in which healthy individuals were instructed to drink alcohol and then undergo polysomnography, it was shown that rapid eye movement (REM) sleep was suppressed during the first half of the sleep cycle followed by an increase during the second half of the sleep cycle. ${ }^{19,27)}$ Alcohol consumption could induce deep sleep at the beginning of the sleep cycle, but if sleep continues for a longer duration, overall sleep quality could deteriorate due to the decreased amount of slow wave sleep and REM sleep rebound. This is in agreement with our findings that, among men, drinking decreased overall sleep duration and increased sleep disturbances.

It has also been reported that alcohol consumption increases sleep apnea ${ }^{28,29)}$ and our study confirmed that number of nighttime awakenings due to snoring was related to alcohol consumption. As mentioned above, alcohol prevents entry into deep sleep and therefore makes a person more susceptible to sleep disturbances given the same conditions. It has also been suggested that alcohol relaxes the upper airway muscles, increasing resistance during inhalation, which directly and significantly impedes breathing. ${ }^{30}$

A number of studies have reported that depression and anxiety deteriorate overall sleep quality. ${ }^{2,4,5,31)}$ Similarly, in this study, we found a significant correlation between anxiety or depression symptoms and deterioration in sleep quality among both men and women. This significant correlation, which remained after excluding persons already diagnosed with anxiety or depression, reaffirms the importance of effective management of depression and anxiety for improving sleep quality.

One limitations of this study is that the results may not reflect the general population because the study only sampled persons who visited a hospital; another limitation is that the number of female subjects with high alcohol consumption levels was low and thus yielded limited statistical significance. We believe that it would be meaningful to conduct further studies on female subjects to compare the sleep quality between hazardous drinkers or patients with alcohol use disorder and moderate drinkers or persons who do not drink at all. In addition, further studies are recommended focused on the correlation between alcohol consumption and sleep apnea or restless leg syndrome, both of which were found to be significant in the present study.

\section{CONFLICT OF INTEREST}

No potential conflict of interest relevant to this article was reported.

\section{REFERENCES}

1. Yang CK. Concise guide to evaluation and management of sleep disor- 
ders. 2nd ed. Seoul: Hana Medical Publishers; 2001.

2. Staner L. Comorbidity of insomnia and depression. Sleep Med Rev 2010; 14:35-46.

3. Buysse DJ. Insomnia. JAMA 2013;309:706-16.

4. Chang KJ, Son SJ, Lee Y, Back JH, Lee KS, Lee SJ, et al. Perceived sleep quality is associated with depression in a Korean elderly population. Arch Gerontol Geriatr 2014;59:468-73.

5. Kenney SR, Paves AP, Grimaldi EM, LaBrie JW. Sleep quality and alcohol risk in college students: examining the moderating effects of drinking motives. J Am Coll Health 2014;62:301-8.

6. Young T, Shahar E, Nieto FJ, Redline S, Newman AB, Gottlieb DJ, et al. Predictors of sleep-disordered breathing in community-dwelling adults: the Sleep Heart Health Study. Arch Intern Med 2002;162:893-900.

7. Aldrich MS, Shipley JE. Alcohol use and periodic limb movements of sleep. Alcohol Clin Exp Res 1993;17:192-6.

8. Yoon IY. Introduction to sleep disorders. Hanyang Med Rev 2013;33: 197-202.

9. Camargo CA Jr, Hennekens CH, Gaziano JM, Glynn RJ, Manson JE, Stampfer MJ. Prospective study of moderate alcohol consumption and mortality in US male physicians. Arch Intern Med 1997;157:79-85.

10. Niroomand F, Hauer O, Tiefenbacher CP, Katus HA, Kuebler W. Influence of alcohol consumption on restenosis rate after percutaneous transluminal coronary angioplasty and stent implantation. Heart 2004;90: 1189-93.

11. Sacco RL, Elkind M, Boden-Albala B, Lin IF, Kargman DE, Hauser WA, et al. The protective effect of moderate alcohol consumption on ischemic stroke. JAMA 1999;281:53-60.

12. Russell M, Chu BC, Banerjee A, Fan AZ, Trevisan M, Dorn JM, et al. Drinking patterns and myocardial infarction: a linear dose-response model. Alcohol Clin Exp Res 2009;33:324-31.

13. Wannamethee G, Shaper AG. Alcohol and sudden cardiac death. $\mathrm{Br}$ Heart J 1992;68:443-8.

14. Corrao G, Bagnardi V, Zambon A, La Vecchia C. A meta-analysis of alcohol consumption and the risk of 15 diseases. Prev Med 2004;38:613-9.

15. Reynolds K, Lewis B, Nolen JD, Kinney GL, Sathya B, He J. Alcohol consumption and risk of stroke: a meta-analysis. JAMA 2003;289:579-88.

16. Min SK. Modern psychiatry. 5th ed. Seoul: Ilchokak; 2009.

17. Cloninger CR. Neurogenetic adaptive mechanisms in alcoholism. Science 1987;236:410-6.

18. Korea Centers for Disease Control and Prevention. Korea National Health and Nutrition Examination Survey (KNHANES V-3) [Internet]. Cheongju: Korea Centers for Disease Control and Prevention; 2012 [cited 2013 Dec 24]. Available from: https://knhanes.cdc.go.kr/knhanes/index.do.

19. Ebrahim IO, Shapiro CM, Williams AJ, Fenwick PB. Alcohol and sleep I: effects on normal sleep. Alcohol Clin Exp Res 2013;37:539-49.

20. Vinson DC, Manning BK, Galliher JM, Dickinson LM, Pace WD, Turner BJ. Alcohol and sleep problems in primary care patients: a report from the AAFP National Research Network. Ann Fam Med 2010;8:48492.

21. Myung SK, Jeong B, Lee WJ, Koh HJ, Suh SY, Yoo T, et al. Standardization of the two-question case-finding instrument as a screening instrument for the adolescent's depression. J Korean Acad Fam Med 2000; 21:100-6.

22. Park HS, Lim JY, Lee SH, Cha YS, Sun WS. Reliability and validity of anxiety screening scale. Korean J Fam Med 2001;22:1224-32.

23. Kim CG, Kim JS, Jung JG, Kim SS, Yoon SJ, Suh HS. Reliability and validity of Alcohol Use Disorder Identification Test-Korean revised version for screening at-risk drinking and alcohol use disorders. Korean J Fam Med 2014;35:2-10.

24. Sohn SI, Kim do H, Lee MY, Cho YW. The reliability and validity of the Korean version of the Pittsburgh Sleep Quality Index. Sleep Breath 2012; 16:803-12.

25. Roehrs T, Roth T. Sleep, sleepiness, and alcohol use. Alcohol Res Health 2001;25:101-9.

26. Chueh KH, Yang MS, Chen CS, Chiou SM. Poor sleep quality and alcohol use problems among elderly Taiwanese aboriginal women. Int Psychogeriatr 2009;21:593-9.

27. Gresham SC, Webb WB, Williams RL. Alcohol and caffeine: effect on inferred visual dreaming. Science 1963;140:1226-7.

28. Aldrich MS, Brower KJ, Hall JM. Sleep-disordered breathing in alcoholics. Alcohol Clin Exp Res 1999;23:134-40.

29. Peppard PE, Austin D, Brown RL. Association of alcohol consumption and sleep disordered breathing in men and women. J Clin Sleep Med 2007;3:265-70.

30. Roth T, Roehrs T, Zorick F, Conway W. Pharmacological effects of sedative-hypnotics, narcotic analgesics, and alcohol during sleep. Med Clin North Am 1985;69:1281-8.

31. Buysse DJ, Angst J, Gamma A, Ajdacic V, Eich D, Rossler W. Prevalence, course, and comorbidity of insomnia and depression in young adults. Sleep 2008;31:473-80. 